\title{
KERNELS OF FREE ABELIAN REPRESENTATIONS OF A LINK GROUP
}

\author{
C. B. SCHAUFELE
}

1. Introduction. Let $L$ denote an oriented link in the 3 -sphere $S^{3}$; let $k_{1}, k_{2}, \cdots, k_{\mu}$ denote the components of $L, G=\pi_{1}\left(S^{3}-L\right)$ the group of the link. $H_{1}\left(S^{3}-L\right)$ is free abelian of rank $\mu$, so we have the exact sequence

$$
0 \rightarrow[G, G] \rightarrow G \rightarrow A_{1} \oplus A_{2} \oplus \cdots \oplus A_{\mu} \rightarrow 0
$$

where $[G, G]$ is the commutator subgroup of $G$ and each $A_{i}, i=1$, $\cdots, \mu$, is infinite cyclic. Thus we have homomorphisms

$$
\phi_{i}: G \rightarrow A_{i}
$$

for each $i=1,2, \cdots, \mu$.

It is the purpose of this paper to determine the structure of the kernels of these homomorphisms. In order to do this, we will construct infinite cyclic covering spaces over $S^{3}-L$ using methods similar to those of Lee Neuwirth in [2]. Using these infinite cyclic covering spaces, we will observe how to construct covering spaces over $S^{3}-L$ corresponding to the kernels of homomorphisms $G \rightarrow A_{i_{1}} \oplus \ldots$ $\oplus A_{i_{m}}, 1 \leqq i_{j} \leqq \mu$, and, under certain conditions, it will be possible to determine the structure of these kernels, in particular, of the commutator subgroup of $G$.

2. Construction of covering spaces. Let $S_{i}^{\prime}$ be an orientable surface in $S^{3}$ which spans the oriented component $k_{i}$ of $L$. We assume $S_{i}^{\prime}$ to be oriented so that $\partial S_{i}^{\prime}=k_{i}$. $S_{i}^{\prime}$ is taken in general position relative to $L-k_{i}$ so that $k_{j} \cap S_{i}^{\prime}, j \neq i$, is a finite set of points. These points are assigned a + or - in the usual manner. If $p_{1}, p_{2} \in k_{j} \cap S_{i}^{\prime}$ have different signs, and if one of the arcs $A \subset k_{j}$ from $p_{1}$ to $p_{2}$ does not intersect $S_{i}^{\prime}$ then we can remove these points of intersection by constructing a new orientable surface $S_{i}^{\prime}$ which spans $k_{i}$ : cut holes in $S_{i}^{\prime}$ at $p_{1}$ and $p_{2}$ and connect the two added boundary curves by a small tube around the arc $A$. Continuing this process, we can remove either all + intersection points, or all - points of intersection of $k_{j}$ with $S_{i}^{\prime}$ without introducing any new points of intersection. We have then an orientable surface $S_{i}^{\prime}$ which spans $k_{i}$ and the number $\eta_{i j}$ of points of intersection of $k_{j}$ with $S_{i}^{\prime}$ is the absolute value of the linking number of $k_{i}$ and $k_{j}$. Repeating this for each $j \neq i$, we ultimately ob-

Received by the editors April 13, 1966. 
tain a surface $S_{i}^{\prime}$ which spans $k_{i}$ and the number of points of intersection of $S_{i}^{\prime}$ with $\bigcup_{j \neq i} k_{j}$ is $\eta_{i}=\sum_{j, j \neq i} \eta_{i j}$. Hence there exists an $S_{i}^{\prime}$ of minimal genus with the above property; if $S_{i}^{\prime}$ is such a surface, let $S_{i}=S_{i}^{\prime}-\bigcup_{j \neq i} k_{j}$. For convenience in this paper, we shall call $S_{i}^{\prime}$ a pre-linking surface and $S_{i}$ a linking surface for $k_{i}$, and call the genus of $S_{i}^{\prime}$ the linking genus of $k_{i}$ with respect to $L$.

Let $U_{i}$ be a regular neighborhood (in the sense of Whitehead [3]) of the linking surface $S_{i}$ for $k_{i}$ such that Bd $U_{i}$ contains $k_{i}$. $\left(\operatorname{Bd} U_{i}\right)-k_{i}$ has two components $S_{i}^{1}$ and $S_{i}^{2}$. Lemmas 1 and 2 in $\$ 4$ of [2] are now valid for $S_{i}^{1}$ and $S_{i}^{2}$.

Lemma 1. The inclusion map $c: S_{i}^{1} \rightarrow \mathrm{Bd} U_{i}$ induces a monomorphism $c_{*}: \pi_{1}\left(S_{i}^{1}\right) \rightarrow \pi_{1}\left(\operatorname{Bd} U_{i}\right)$.

Lemma 2. The inclusion map $d: S_{i}^{1} \rightarrow S^{3}-\left(\operatorname{Int}\left(U_{i}\right) \cup L\right)$ induces a monomorphism $d_{*}: \pi_{1}\left(S_{i}^{1}\right) \rightarrow \pi_{1}\left(S^{3}-\left(\operatorname{Int}\left(U_{i}\right) \cup L\right)\right)$.

Some remarks on the proofs of these two lemmas are perhaps in order. In the proof of Lemma $1, k_{i}$ may be unknotted in which case $S_{i}$ may be a disk. However, assuming $L$ is not splittable, $S_{i}^{1}$ must be a disk with a number of points removed, so that $k_{i} \rightarrow S_{i}^{1}$ still induces a monomorphism. In the proof of Lemma 2, by assuming there is a closed curve $\alpha$ on $S_{i}^{1}$ such that $\alpha \cong 0$ in $S^{3}-\left(\operatorname{Int}\left(U_{i}\right) \cup L\right)$ but not on $S_{i}^{1}$, one either reduces the genus of $S_{i}^{\prime}$ or reduces the number $\eta_{i}$.

For each $i=1,2, \cdots, \mu$, let $H_{i}$ be the normal subgroup of $G$ consisting of all elements of $G$ which are represented by loops in $S^{3}-L$ which have linking number zero with $k_{i}$. Clearly $G / H_{i}=A_{i}$ so that the kernel of $\phi_{i}$ is $H_{i}$.

Using the linking surface $S_{i}$ for $k_{i}$, we can now construct the covering space $X_{i}$ of $S^{3}-L$ corresponding to $H_{i}=\operatorname{ker} \phi_{i}$. This is done just as in [2], but we include a brief description of $X_{i}$ for completeness.

Let $Y_{0}^{i}=S^{3}-\left(\operatorname{Int}\left(U_{i}\right) \cup L\right)$, and let $\left\{Y_{j}^{i}\right\}_{j=-\infty}^{\infty}$ be a countable collection of disjoint copies of $Y_{0}^{i}$. Each $Y_{j}^{i}$ has two boundary components ${ }_{j} S_{i}^{1}$ and ${ }_{j} S_{i}^{2}$ which are homeomorphic in a natural way to ${ }_{0} S_{t}^{1}=S_{i}^{1}$ and ${ }_{0} S_{i}^{2}=S_{i}^{2}$, respectively. Also $S_{i}^{1}$ and $S_{i}^{2}$ are homeomorphic, so we construct $X_{i}$ by attaching $Y_{j}^{i}$ to $Y_{j+1}^{i}$, glueing ${ }_{j} S_{i}^{1}$ to ${ }_{j+1} S_{i}^{2}$ for each $j$, $-\infty<j<\infty$.

Clearly $\pi_{1}\left(Y_{0}^{i}\right) \subset H_{i}$, and since $G / H_{i}$ is infinite cyclic, it follows that $X_{i}$ is the covering space of $S^{3}-L$ corresponding to $H_{i}$.

3. Group structure. The proof of Theorem 1 of [2] with $[G, G]$ replaced by $H_{i}$ is valid. Thus we have:

THEOREM 3.1. If $H_{i}$ is finitely generated, it is free of rank $2 g_{i}+\eta_{i}$ 
where $g_{i}$ is the linking genus of $k_{i}$ with respect to $L$, and $\eta_{i}$ is the sum of the absolute values of the linking numbers of $k_{i}$ with $k_{j}, j \neq i$.

If $H_{i}$ is not finitely generated, then either it is

(a) a nontrivial free product with amalgamation on a free group $F$ of rank $2 g_{i}+\eta_{i}$,

$$
\cdots * A \underset{F}{*} A \underset{F}{*} A * \cdots
$$

or,

(b) locally free and a direct limit of free groups of rank $2 g_{i}+\eta_{i}$.

The following lemma will be used in the sequel.

Lemma 3.2. (i) $\bigcap_{i=1}^{k-1} H_{i} / \bigcap_{i=1}^{k} H_{i}=A_{k}, 2 \leqq k \leqq \mu$.

(ii) $G / \bigcap_{i=1}^{k} H_{i}=A_{1} \oplus A_{2} \oplus \cdots \oplus A_{k}, 1 \leqq k \leqq \mu$.

Proof. We prove (i). (ii) is proved in a similar manner. For each $i=1,2, \cdots, \mu, G \supset H_{i} \supset[G, G]$ since $G / H_{i}$ is abelian. Thus

$$
G /[G, G]=A_{1} \oplus \cdots \oplus A_{\mu} \supset \bigcap_{i=1}^{m} H_{i} /[G, G]=A_{m+1} \oplus \cdots \oplus A_{\mu}
$$

so that

$$
\begin{aligned}
\bigcap_{i=1}^{k-1} H_{i} / \bigcap_{i=1}^{k} H_{i} & =\left(\bigcap_{i=1}^{k-1} H_{i} /[G, G]\right) /\left(\bigcap_{i=1}^{k} H_{i} /[G, G]\right) \\
& =\left(A_{k} \oplus A_{k+1} \oplus \cdots \oplus A_{\mu}\right) /\left(A_{k+1} \oplus \cdots \oplus A_{\mu}\right) \\
& =A_{k} .
\end{aligned}
$$

We will now restrict our attention to the situation when $\mu=2$ and the linking number of $k_{1}$ and $k_{2}$ is nonzero; i.e., $\eta_{12}=\eta_{21}=\eta_{1}=\eta_{2} \neq 0$. The covering space $X_{12}$ of $S^{3}-L$ corresponding to $H_{1} \cap H_{2}$ will be constructed and, from this, the structure of $H_{1} \cap H_{2}$ can be determined. Note that by Lemma 3.2, $H_{1} \cap H_{2}=\operatorname{ker}\left[G \rightarrow A_{1} \oplus A_{2}\right]=[G, G]$.

Before constructing $X_{12}$, we need three lemmas. Let $S_{1}^{\prime}, S_{2}^{\prime}$, and $S_{1}, S_{2}$ be pre-linking and linking surfaces for $k_{1}, k_{2}$, respectively. We have already constructed the covering space $X_{1}$ of $S^{3}-L$ corresponding to $H_{1}$. Let $p_{1}: X_{1} \rightarrow S^{3}-L$ be the covering map.

Lemma 3.3. $p_{1}^{-1}\left(S_{2}\right)$ is a connected orientable 2-manifold in $X_{1}$.

Proof. Since $\eta_{12} \neq 0, k_{2}$ intersects $S_{1}^{\prime}$. Intersections of $S_{1}^{\prime}$ and $S_{2}^{\prime}$ are either simple closed curves which lie on the interiors of $S_{1}^{\prime}$ and $S_{2}^{\prime}$, or arcs which run from $k_{1}$ to $k_{2}$ (no arc can run from $k_{i}$ to $k_{i}$ by the definition of $S_{1}^{\prime}$ and $S_{2}^{\prime}$ ). It follows from this that all the com- 
ponents of $p_{1}^{-1}\left(k_{2}\right)$ lie on the same component of $p_{1}^{-1}\left(S_{2}\right)$. Hence, by the path lifting property, $p_{1}^{-1}\left(S_{2}\right)$ is connected.

Lemma 3.4. The inclusion $i: p_{1}^{-1}\left(S_{2}\right) \rightarrow X_{1}$ induces a monomorphism $i^{*}: \pi_{1}\left(p_{1}^{-1}\left(S_{2}\right)\right) \rightarrow \pi_{1}\left(X_{1}\right)$.

Proof. Since $p_{1}^{-1}\left(S_{2}\right)$ is connected, it is a covering space over $S_{2}$ with covering map $q=p_{1} \mid p_{1}^{-1}\left(S_{2}\right)$.

The diagram

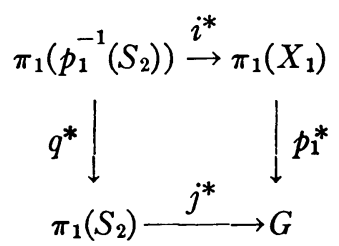

is commutative, $j^{*}$ is induced by inclusion. $q^{*}, j^{*}$, and $p_{1}^{*}$ are all monomorphisms, and hence so is $i^{*}$.

Lemma 3.5. $X_{1}-p_{1}^{-1}\left(S_{2}\right)$ is connected, and

$$
h^{*}\left(\pi_{1}\left(X_{1}-p_{1}^{-1}\left(S_{2}\right)\right) \subset\left(p_{1}^{*}\right)^{-1}\left(H_{1} \cap H_{2}\right) \subset \pi_{1}\left(X_{1}\right) \approx H_{1},\right.
$$

where $h^{*}$ is induced by the inclusion map $h: X_{1}-p_{1}^{-1}\left(S_{2}\right) \rightarrow X_{1}$.

Proof. $X_{1}-p_{1}^{-1}\left(S_{2}\right)$ is connected since $p_{1}^{-1}\left(S_{2}\right)$ is a connected 2 manifold with boundary $p_{1}^{-1}\left(k_{2}\right)$.

$p_{1} h$ maps $X_{1}-p_{1}^{-1}\left(S_{2}\right)$ onto $S^{3}-\left(L \cup S_{2}\right)$, so $p_{1}^{*} h^{*}\left(\pi_{1}\left(X_{1}-p_{1}^{-1}\left(S_{2}\right)\right)\right)$ $\subset \mathrm{H}_{2}$.

Now, by Lemma 3.2,

$$
\pi_{1}\left(X_{1}\right) /\left(p_{1}^{*}\right)^{-1}\left(H_{1} \cap H_{2}\right) \approx H_{1} /\left(H_{1} \cap H_{2}\right)=A_{2},
$$

so we can construct the infinite cyclic covering space $X_{12}$ over $X_{1}$ corresponding to $\left(p_{1}^{*}\right)^{-1}\left(H_{1} \cap H_{2}\right)$ with covering map $p_{12}^{\prime}$. This is done just as $X_{1}$ was constructed, with $p_{1}^{-1}\left(S_{2}\right)$ replacing $S_{1}$. Thus $X_{12}$ is the covering space of $S^{3}-L$ corresponding to $H_{1} \cap H_{2}=[G, G]$ with covering map $p_{12}=p_{1} p_{12}^{\prime}$.

Note that by the method of construction of $X_{1}, \pi_{1}\left(p_{1}^{-1}\left(S_{2}\right)\right)$ is either trivial, or free and infinitely generated; so by Lemma 3.4 and the proof of Theorem 1 of [2], we have:

THEOREM 3.6. If $G$ is not $Z \oplus Z,[G, G]$ is infinitely generated and has one of the following forms:

(a) free;

(b) an infinite free product of isomorphic groups;

(c) an infinite free product of isomorphic groups with amalgamation 
on an infinitely generated free group;

(d) locally free and a direct limit of infinitely generated free groups.

When $\mu>2$ and $\eta_{i j} \neq 0(i \neq j)$ for each $i, j, 1 \leqq i \leqq \mu, 1 \leqq j \leqq \mu$, the above method can be used to construct the covering space of $S^{3}-L$ corresponding to $\operatorname{ker}\left[G \rightarrow A_{i_{1}} \oplus \cdots \oplus A_{i_{m}}\right]$ so that Theorem 3.6 is valid with $[G, G]$ replaced by this subgroup. For, by reordering the components of $L$, we can assume that the homomorphism in question is $G \rightarrow A_{1} \oplus A_{2} \oplus \cdots \oplus A_{m}$, so that by Lemma 3.2, the kernel of the homomorphism is $\bigcap_{i=1}^{m} H_{i}$. Thus, we can construct a finite number of covering spaces $X_{12} \ldots m, X_{12} \ldots(m-1), \ldots, X_{1}$ of $S^{3}-L$ such that $X_{12} \ldots K$ corresponds to $\bigcap_{i=1}^{k} H_{i}$. If $p_{12} \ldots k: X_{12} \ldots k \rightarrow S^{3}-L$ is the covering map, $X_{12} \ldots(k+1)$ is the infinite cyclic covering space of $X_{12} \ldots k$ corresponding to $\left(p_{12}^{*} \cdots \mathbf{k}\right)^{-1}\left(\bigcap_{i=1}^{k+1} H_{i}\right) . X_{12} \cdots(k+1)$, is of course constructed as $X_{12}$ was constructed above, replacing $p_{1}^{-1}\left(S_{2}\right)$ by $p_{12}^{-1} \ldots k\left(S_{k+1}\right)$. The requirement that $\eta_{i j} \neq 0$ insures that $p_{12}^{-1} \ldots k\left(S_{k+1}\right)$ will be connected for each $k=1,2, \cdots, \mu-1$. This allows the use of the Van Kampen theorem, in the proof of Theorem 3.6. However, should $\eta_{i j}=0$ for some $i$ and $j$, the covering spaces can still be constructed even though $p_{12}^{-1} \ldots_{\mathbf{k}}\left(S_{k+1}\right)$ will not necessarily be connected.

The following theorem gives a sufficient condition for $[G, G]$ to be a free product.

THEOREM 3.7. If there is a component $k_{2}$ of $L$ such that $\eta_{2}=1$ and the linking genus $g_{2}$ of $k_{2}$ is zero, and if $G \neq Z \oplus Z$, then $[G, G]$ is a free product.

PRoof. Since $\eta_{2}=1$ and $g_{2}=0$, the linking surface $S_{2}$ of $k_{2}$ is a "half open" annulus. Let $k_{i}$ be the component of $L$ such that $\eta_{i 2}=1$ and let $S_{i}$ be its linking surface. If we assume $i=1$, we can construct the covering spaces $X_{1}$ and $X_{12}$. Since $\eta_{12} \neq 0, p_{1}^{-1}\left(S_{2}\right)$ is connected and hence is a covering space over $S_{2}$. Now $p_{1}^{*} \pi_{1}\left(p_{1}^{-1}\left(S_{2}\right)\right) \subset H_{1} \cap \pi_{1}\left(S_{2}\right)$, and since $\eta_{12}=1, \pi_{1}\left(S_{2}\right) \cap H_{1}=0$. Hence $\pi_{1}\left(p_{1}^{-1}\left(S_{2}\right)\right)$ is trivial. Since $H_{1} \cap H_{2}$ is a free product with amalgamation on $\pi_{1}\left(p_{1}^{-1}\left(S_{2}\right)\right), H_{1} \cap H_{2}$ is just a free product. $H_{1} \cap H_{2} \supset[G, G]$ and so by the Kurosh Subgroup Theorem $[1],[G, G]$ is a free product.

\section{REFERENCES}

1. A. G. Kurosh, Theory of groups, Vol. II, Chelsea, New York, 1960.

2. L. Neuwirth, The algebraic determination of the genus of knots, Amer. J. Math. 82 (1960), 791-798.

3. J. H. C. Whitehead, Simplicial spaces, nuclei, and m-groups, Proc. London Math. Soc. 45 (1939), 243-327.

The University of Georgia 